\title{
Study on Adaptability of Lateral Concentrated Smoke Exhausting Mode in Large-span Rectangular Tunnels
}

\author{
Zhengmao CAO ${ }^{1,}$ a , Xiao LIU ${ }^{2, b}$
}

${ }^{1}$ China Merchants Chongqing Communications Research and Design Institute Co., Ltd., Chongqing
400067, China

${ }^{2}$ Department of foreign language, Southwest Jiaotong University Hope College, Chengdu 610400, China

aswjtuczm@163.com, bxiaoliu13@foxmail.com

Keywords: Large-span tunnel, rectangular tunnel, lateral concentrated exhaust, numerical simulation, adaptability of smoke exhausting

Abstract. Taking large-span rectangular underwater tunnel as the research objective, numerical calculation model of lateral concentrated smoke exhausting in rectangular underwater tunnel was established by FDS. The adaptability of lateral concentrated exhaust mode in tunnels of variable widths is obtained according to analysis of smoke distribution and smoke exhausting efficiency under different working conditions. The results are as follows: The increase of width results in the decrease of smoke exhausting efficiency. In the two-lane, uni-directional tunnel, the smoke exhausting efficiency is $83.38 \%$; while that of six-lane, uni-directional tunnel is only $55.19 \%$. The wind velocity is higher in the area near the lateral exhaust valves; and which is lower in the area far from the exhaust valves. The influence of wind velocity for lateral exhaust valves is limited. With the increase of width, the wind velocity of cross-section decreases dramatically. In a two-lane, uni-directional tunnel, the wind velocity of the right wall decreases by $32.03 \%$, and that in six-lane, uni-directional tunnel decreases by $59.62 \%$. For large-span rectangular tunnels of uni-directional four-lane or even wider ones, the lateral centralized exhaust mode shows poor adaptability.

\section{Introduction}

Recently the longitudinal ventilation is the most commonly used smoke exhausting mode in tunnel fire. It can effectively prevent the smoke from flowing reversely and exhaust smoke from the tunnel. However, the longitudinal ventilation doesn't work well when the traffic is blocked in tunnel fire. In this case, the concentrated smoke exhaust mode is applied. The lateral and top exhaust valves around the fire source are opened to discharge smoke. Since the concentrated smoke exhausting mode can effectively control the spread of smoke within tunnels, it is commonly used in major projects like underwater tunnels and urban tunnels.

Domestic and foreign scholars have conducted many studies concerning roof ventilation and achieved much progress. R.K. Harish studied the effects of buoyancy induced roof ventilation system on the characteristics of fire with different numbers of unclosed exhaust valves [1]. O. Vauquelin carried out smoke extraction experiments [2]. Y.H. QIU et al. conducted many studies on roof concentrated smoke exhausting mode [3,4].

Only a few researches focus on lateral concentrated smoke exhausting mode. Y. LI carried out numerical simulation calculation on efficiency and output of smoke exhausted in two-lane tunnel with lateral valves [5]. The submarine tunnel of HZM adopts the lateral smoke exhaust mode. $\mathrm{H}$. Wu conducted optimization research on the size of its exhaust valves [6] and K. Tian carried out smoke exhausting experiment in this submarine tunnel [7]. In terms of large-span tunnel fire, few researches are concerned about the efficiency and adaptability of lateral concentrated exhaust mode. Moreover, the studies on characteristics of fire in tunnels of different width adopting lateral smoke exhausting mode are still lacked both at home and abroad. Therefore, it is necessary to conduct studies on the adaptability of lateral exhaust mode in large-span rectangular tunnel, which can provide theoretical support for the design of smoke exhausting in similar projects. 


\section{Project Overview}

The research is based on a $6845 \mathrm{~m}$ long underwater tunnel project, and the cross section is large-span adopting "two tunnels with one pipe gallery" design. The upper part of the pipe gallery is an exclusive longitudinal smoke exhausting channel. Smoke is exhausted outside from lateral smoke exhausting valves to exclusive longitudinal smoke exhausting channel during tunnel fire. The net width of standard cross section for single tunnel is $18.3 \mathrm{~m}$, and its net height of $7.6 \mathrm{~m}$. The widest section of the main tunnel interchange area is a uni-directional six-lane, which is $27.99 \mathrm{~m}$ wide. Fig. 1 and Fig. 2 illustrate the cross section of the tunnel.

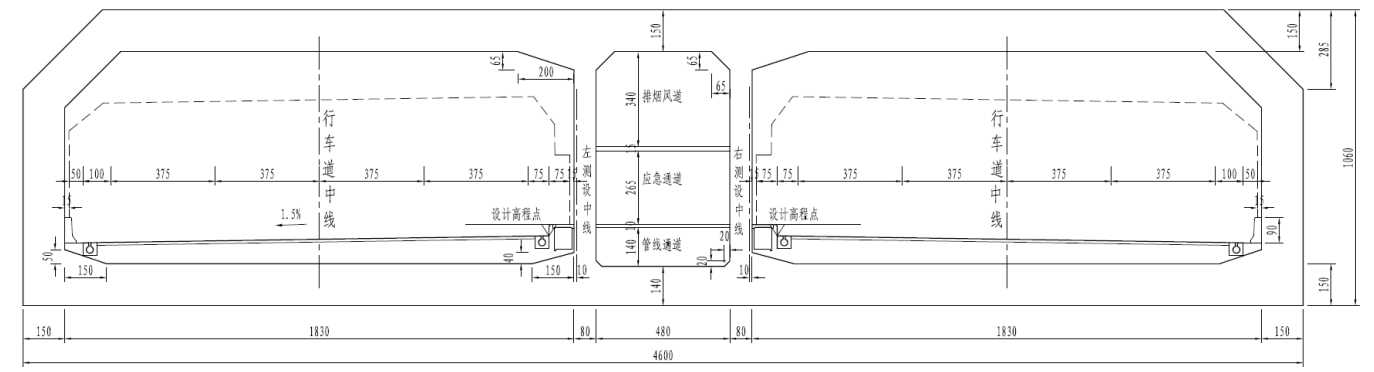

Fig. 1 Standard Cross Section of Main Tunnel

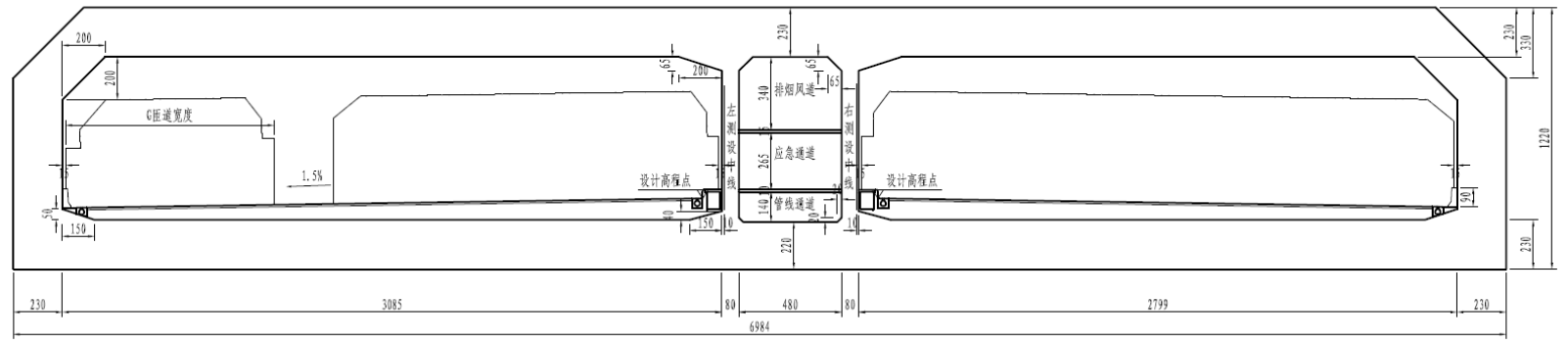

Fig. 2 Widened Cross Section of Main Tunnel Interchange Area

The tunnel is characterized by a large-span cross section, and the structure is flattened severely which increases the difficulty of ventilation and smoke exhausting during a tunnel fire. According to the characteristics of lateral concentrated smoke exhausting in large span rectangular tunnel, numerical simulation of fire in case of different lanes is conducted to analyze the applicability of the lateral concentrated smoke exhausting method in the large span rectangular tunnel.

\section{Calculating Model and Parameter Setting}

\section{Establishment of Model}

The numerical calculation model is established by FDS according to cross-section dimensions of actual tunnel. It is assumed that only a fire happens at a time and the smoke doesn't spread to the adjacent tunnel. Therefore, the simulation is limited to the tunnel where the fire happened. Smoke exhausting valves connect the main tunnel and longitudinal smoke exhausting channel. Each valve is $3 \mathrm{~m}$ wide and $2 \mathrm{~m}$ high, and the upper side is $0.3 \mathrm{~m}$ away from the top board of the tunnel. The distance between each valve is $60 \mathrm{~m}$. Fig. 3 illustrates the layout of smoke exhausting valves. 


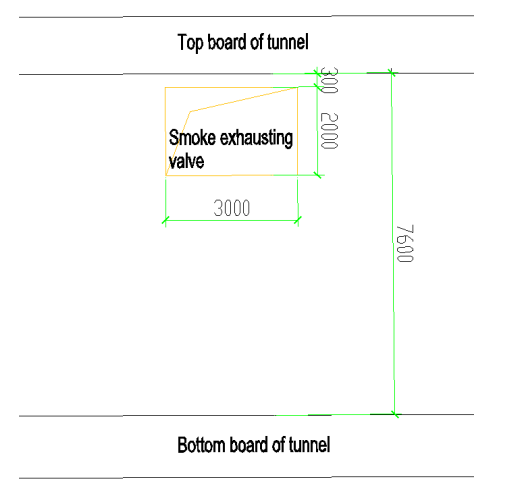

(a)Size of smoke exhausting valves(mm)

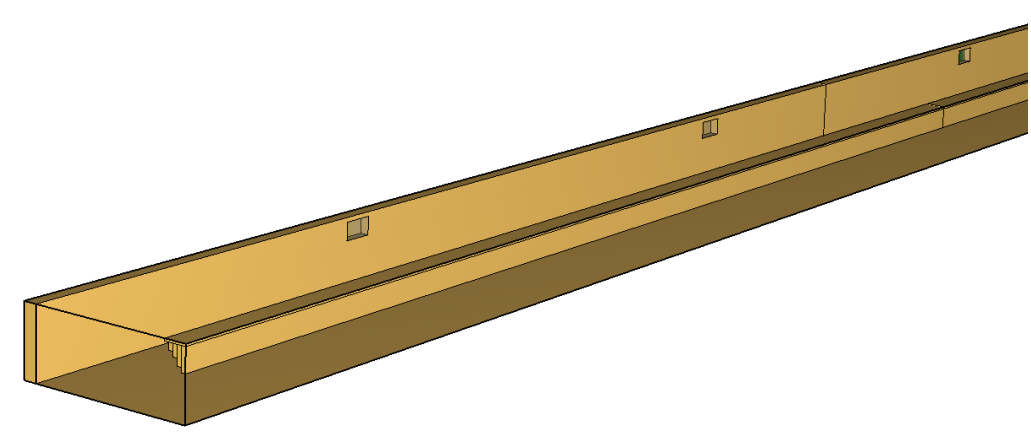

(b)Layout of lateral smoke exhausting valves(Longitudinal)

Fig. 3 Layout of Lateral Exhaust Valves in Tunnel

During the fire, the temperature of smoke and concentration of harmful gas around the fire source change dramatically. According to previous research results, smoke exhausting valves within 300m of the fire source are opened to exhaust smoke. Considering the influence of boundary condition on flow field in tunnel, the numerical calculation model for tunnel of 1000 meters long is established. Fire is usually caused by large vehicles, therefore, the fire source at the cross section of the tunnel is placed in the right lane according to the direction of traffic, and in the center of the tunnel longitudinally.

\section{Grid Setting}

The accuracy of fire numerical simulation calculation increases with the decrease of the grid size. A lot of researches prove that the grid size should be $10 \%$ of fire characteristic diameter in numerical simulation calculation. Since there is high temperature gradient near the fire source, the grid at $y$ direction within $50 \mathrm{~m}$ of the fire source is $0.25 \mathrm{~m}$, while that at other areas is $0.5 \mathrm{~m}$. The model grid at $\mathrm{x}$ direction and $\mathrm{z}$ direction is $0.25 \mathrm{~m}$.

\section{Parameters Setting}

The numerical calculation mainly studies the influence of different widths on the efficiency of lateral concentrated smoke exhausting in tunnel fire with the main focus on the maximum HRR stage of fire. The fuel is set as heptane, and its burning characteristic is default value in FDS database. The maximum HRR is set as 50MW. Considering the air leakage of smoke exhausting system and the loss of resistance along the tunnel, the minimum volume of exhausting smoke is $189 \mathrm{~m}^{3} / \mathrm{s}$. According to previous tunnel fire, the burning face of fire source is $1 \mathrm{~m}$ high above the floor and the area is set as 25 $\mathrm{m}^{2}$, the projected area of large vehicle. Four exhausting valves near the fire source were opened to exhaust smoke 90 s after the fire.

\section{Calculating Model of Smoke Exhausting Efficiency}

The smoke exhausting efficiency is the percentage of the smoke exhausted from the exhausting valve per unit time to the total amount of smoke generated by the fire, the amount of smoke is represented by the amount of CO. Smoke exhausting efficiency calculation formula is as follows:

$$
\eta=\frac{\sum m_{C O-e i}}{m_{C O-p}} \times 100 \%
$$

Where $\eta$ is the smoke exhausting efficiency, $\% ; m_{\mathrm{CO}-\mathrm{e} i}$ is the smoke exhausting amount of No.i exhausting valve, $\mathrm{kg} / \mathrm{s} ; m_{\mathrm{CO}-\mathrm{p}}$ is the total amount of smoke generated by the fire $\mathrm{kg} / \mathrm{s}$. 
Under the 50MW fire condition, the CO production per unit time is $6.579 \mathrm{~g} / \mathrm{s}$ theoretically. The exhausted amount of $\mathrm{CO}$ from each exhausting valve could be obtained by numerical simulation.

\section{Result and Analysis}

When the total volume of exhausting smoke is $189 \mathrm{~m}^{3} / \mathrm{s}$ and the maximum HRR is 50MW, numerical simulation of fire is carried out for tunnels with different lanes, which included uni-directional two-lane(width $10.5 \mathrm{~m}$ ), uni-directional three-lane(width $14.4 \mathrm{~m}$ ), uni-directional four-lane(width $18.3 \mathrm{~m}$ ), uni-directional five-lane(width $23.1 \mathrm{~m}$ ), uni-directional six-lane(width $28 \mathrm{~m}$ ). The smoke diffusion in the tunnel is shown in Figure 4.

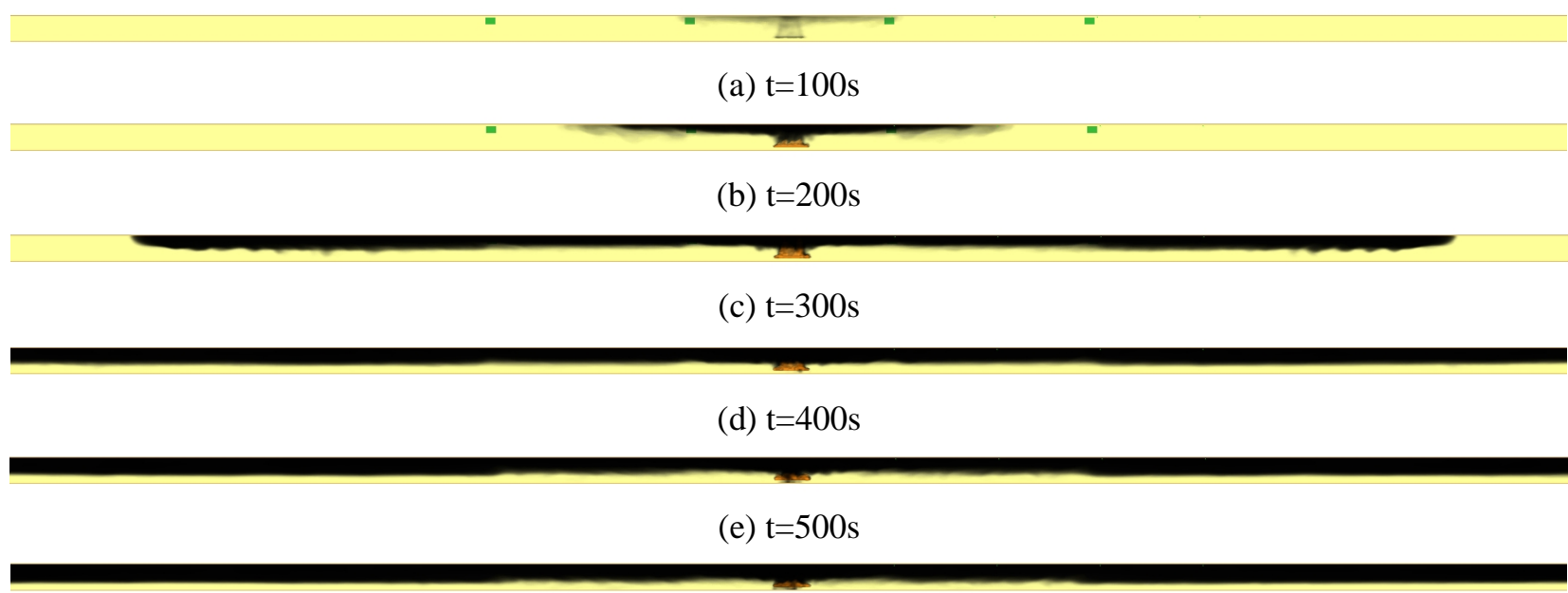

(f) $t=600 \mathrm{~s}$

Fig. 4 Diffusion of Smoke in Tunnel

\section{Smoke exhausting efficiency under different widths of tunnel}

According to the calculation method of smoke exhausting efficiency, calculation result of rectangular tunnel with different widths adopting lateral concentrated smoke exhausting method is obtained, as is shown in Figure 5.

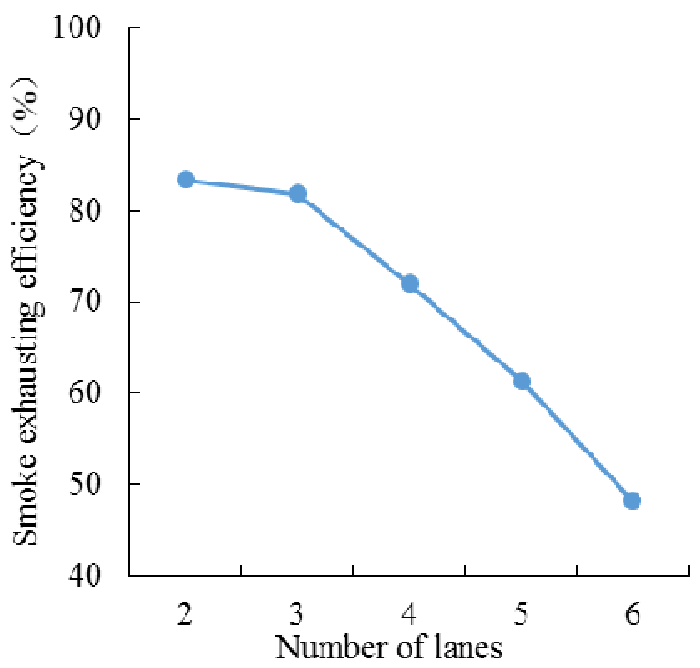

Fig. 5 Smoke Exhausting Efficiency under Different Widths of Tunnel

According to the calculation results, the increase of the width in tunnel results in the decrease of smoke exhausting efficiency. The smoke exhausting efficiency of two-lane, uni-directional tunnel is $83.38 \%$; there is small change in uni-directional three-lane tunnel with efficiency of $81.84 \%$. When the 
width of single tunnel exceeds four lanes, the efficiency decreased dramatically. The smoke exhausting efficiency of uni-directional four-lane tunnel is $72.03 \%$, while that of six-lane, uni-directional one is only $48.19 \%$.

\section{Characteristics of Wind Velocity Distribution in Tunnels of Different Widths}

The calculation results of smoke exhausting efficiency change significantly in tunnels of different widths. One of the main reasons is that the smoke exhausted in different tunnels is limited with the increase of the width. Therefore, it is necessary to conduct research on flow field of wind velocity in tunnel. As is shown in Figure 6.

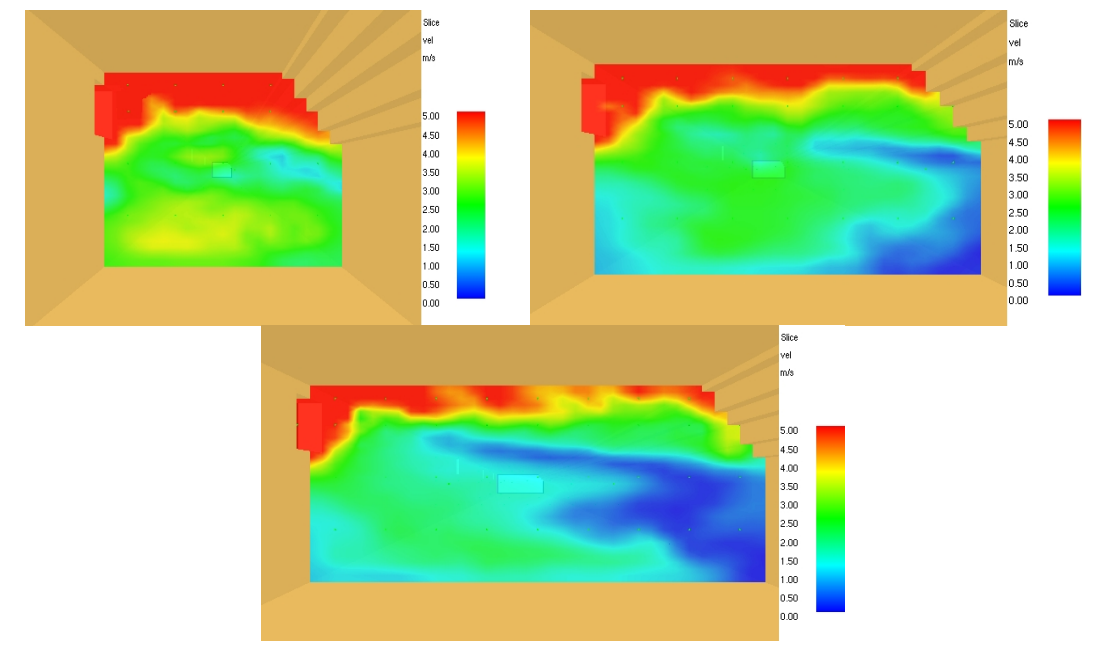

(a)two-lane tunnel

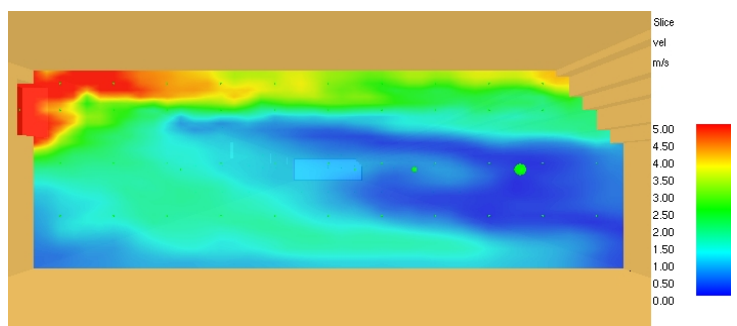

(d)five-lane tunnel

(b)three-lane tunnel

(c)four-lane tunnel

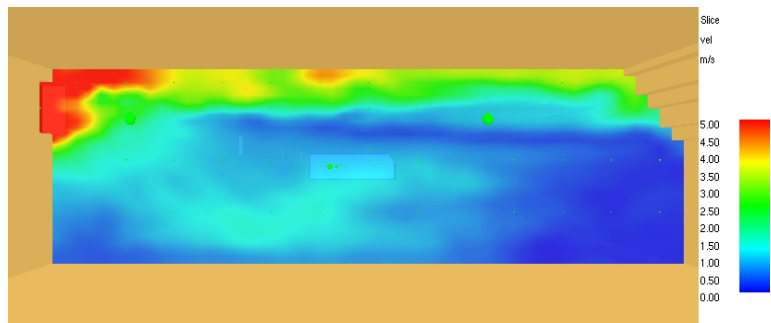

(e)six-lane tunnel

Fig. 6 Velocity Distribution under Different Widths of Tunnel

According to the cross-section wind velocity distribution of different tunnel widths, the wind velocity is higher near the smoke exhausting valve area at the left wall of tunnel. There is little change in the cross-section distribution of wind velocity in tunnels of smaller width. Because the influence of lateral exhaust valves on wind velocity is limited, there is obviously attenuation of wind velocity far from the valves, especially nearby the right wall of tunnel. The cross-sectional wind velocity of the same height near the right wall is analyzed and the calculation results are illustrated in Fig.7 and Fig.8. 


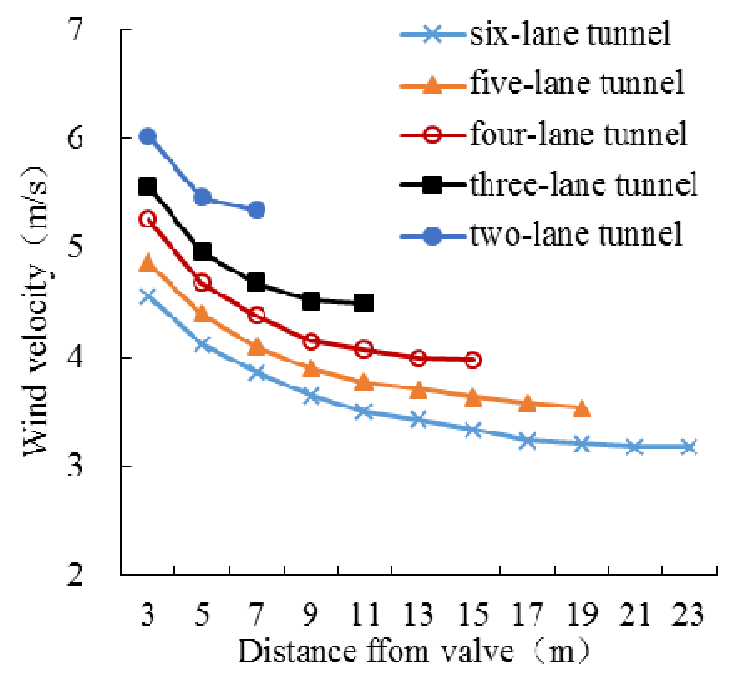

Fig. 7 Wind Velocity of Cross-section in tunnel

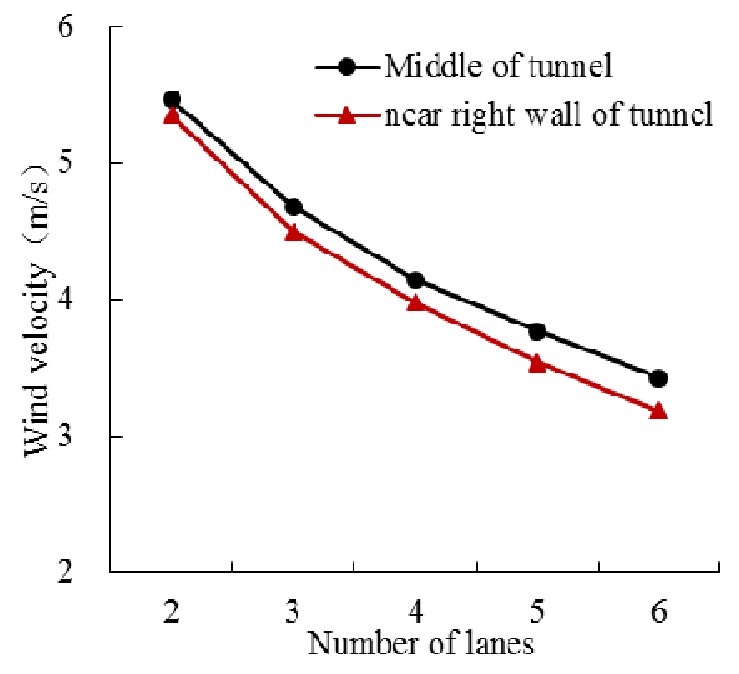

Fig. 8 Wind Velocity under Different Widths of Tunnel

The wind velocity gradually decreases in tunnels with the increase of distance from the valves. The cross-sectional wind velocity in narrower tunnel is larger than that of wider ones. The wind velocity in the middle of uni-directional two-lane tunnel is $5.47 \mathrm{~m} / \mathrm{s}$ in; while that of four-lane tunnel is $4.15 \mathrm{~m} / \mathrm{s}$ and the six-lane one is $3.43 \mathrm{~m} / \mathrm{s}$.

The wind velocity near the right wall of the tunnel is analyzed and the results are as follows. The wind velocity in this area of uni-directional two-lane tunnel is $5.35 \mathrm{~m} / \mathrm{s}$, with an attenuation of $32.03 \%$ compared with that of lateral valves. That of uni-directional four-lane tunnel is $3.98 \mathrm{~m} / \mathrm{s}$, with an attenuation of $49.46 \%$. In uni-directional six-lane tunnel, the wind velocity is $3.18 \mathrm{~m} / \mathrm{s}$, with an attenuation of $59.62 \%$.

According to the calculation results of the cross-sectional wind velocity flow field in tunnels of different widths, the increase of the tunnel width has minor influence on the wind velocity near the smoke exhausting valves, but the influence is obvious near the right wall. Because of the limited influence of lateral exhausting valves on wind velocity, and the attenuation of wind velocity in right area is severely when tunnel is too wide. It results in the smoke accumulation near the right wall of tunnel. Moreover, the high temperature and concentration of harmful gas severely affect the efficiency of smoke exhausting.

\section{Conclusions}

The fire numerical simulation calculation is conducted in rectangular tunnels of variable widths under lateral concentrated smoke exhausting mode. Meanwhile, it also studies the smoke exhausting efficiency and distribution of wind velocity flow field under different working conditions. The results are as follows:

(1) The increase of width results in the decrease of smoke exhausting efficiency. The smoke exhausting efficiency of two-lane uni-directional tunnel is $83.38 \%$; while that of four-lane uni-directional tunnel is $72.03 \%$. The efficiency of six-lane uni-directional tunnel is only $55.19 \%$.

(2) The influence of lateral valves wind velocity is limited. With the increase of tunnel width, the cross-sectional wind velocity decreases dramatically. The right wall area of two-lane uni-directional tunnel decreases by $32.03 \%$; while that of four-lane uni-directional tunnel decreases by $49.46 \%$ and the six-lane uni-directional tunnel decreases by $59.62 \%$.

(3) For uni-directional tunnels of two lanes and three lanes, the lateral concentrated smoke exhausting mode works well. For large-span rectangular tunnels of uni-directional four-lane or even wider tunnel, the lateral centralized exhaust mode shows poor adaptability. It is suggested that additional smoke exhausting measures should be adopted in smoke exhausting design for large-span rectangular tunnel fire to better the smoke exhausting efficiency. 


\section{References}

[1] R.K. Harish: submitted to Tunnelling and Underground Space Technology (2014).

[2] O. Vauquelin and O. Meget: submitted to Fire Safety Journal (2002).

[3] Y.H. Qiu, B. Lou, J.H. Xu and X.F. Long: submitted to Journal of Safety and Environment (2016).

[4] Y.P. Pan, Z.S Xu, S. Wang and D.X. Wu: submitted to Chinese Journal of Underground Space and Engineering (2012).

[5] Y.L and S.J. Cai: submitted to Journal of Dalian Jiaotong University (2016).

[6] H. Wu: Optimization Research on Smoke Vent Size of HZM Submarine Tunnel under Fire Case (Chang' an University, China 2010).

[7] K. Tian: Experimental Research on Fire Smoke Exhaust in Extra Long Immersed Tunnel (Chongqing Jiaotong University, China 2015). 